richts in anderen Staaten. Andererseits vermitteln die Exkursionen wertvolle Anschauung über Land und Leute. Ein weiterer Vorzug internationaler Veranstaltungen besteht im Gedankenaustausch mit Vertretern anderer Staaten, wozu sich im Verlaufe dieser Studienwoche genug Gelegenheit bot.

Die nächste internationale Tagung soll von den Deutschen Schulgeographen übernommen und möglicherweise im Rheinland durchgeführt werden.

\title{
DIE SCHWEIZ 1957 EIN LANDESKUNDLICHER RÜCKBLICK
}

\section{P. KöCHLI}

Witterungsverlauf. Durchschnittlich wichen Temperatur, Niederschläge und Sonnenscheindauer wenig von den langjährigen Mittelwerten ab.

Auf der Alpennordseite war der Januar zu kalt und zu trocken, der Februar war mild und naß, der März der mildeste der bisherigen Meßreihe überhaupt, so daß in einzelnen Gegenden Kirschen und Birnen bereits Ende März blühten. Auch der April war zu warm, der Mai hingegen zu kalt und zu trocken. Katastrophal wirkte sich die Frostnacht vom 7./8. Mai mit Minustemperaturen von $7^{\circ}$ bis $9^{\circ} \mathrm{C}$ aus. Der Juni brachte nach heftigen Föhntagen (kräftige Schneeschmelze) außerordentlich große Niederschläge, die im Nikolaital (Täsch) und im Bündnerland zu schweren Verwüstungen führten. Juli, August, September waren mit Ausnahme von ein paar heißen und trockenen Tagen zu Julibeginn zu kalt und hatten überdurchschnittlich viele Niederschlagstage. Oktober, November und Dezember waren etwas zu warm und zu trocken. - Die Alpensüdseite zeigte dagegen fast normale Verhältnisse. Schwere Hagelschläge traten zwischen dem 21. und 26. Juni auf und führten zu 757o Schadenanzeigen aus 343 Gemeinden, insbesondere aus Lavaux, Gruyère, Sensebezirk, Schwarzenburg, Seftigen, Konolfingen, Signau, Sursee, Willisau, Zürcher Oberland, unteres Toggenburg, Neuenburgerjura; im August vor allem aus dem Mendrisiotto und der Magadinoebene usw. Insgesamt wurden aus 1084 Gemeinden 19589 Schadenmeldungen (9,06 Mio Franken) eingereicht. Auf das Wettergeschehen sind zahlreiche Überschwemmungen und Rutschungen zurückzuführen, so im Februar zwischen Erlinsbach und Kienberg, bei Täsch und im Bündnerland im Juni, im August bei Amsteg, Seewis-Valzeina im Prätigau, am Lauigraben bei Oberwil i. S., bei St-Ursanne (Montmelon), bei Bodio-Fiesso usw. Auch die bekannten Rutschungen bei Peiden und Schuders verstärkten sich.

Wirtschaft. Landwirtschaftlich war 1957 sehr wechselvoll. Bei Obst, Reben und Gemüsen machten sich die Maifröste teilweise verheerend bemerkbar. Beim Obst wurde etwa ein Zehntel einer Mittelernte gewonnen, bei den Reben war der Ertragsausfall in einzelnen Gebieten, hauptsächlich in der Ostschweiz, vollständig. Gute Ernteerträge verzeichneten hingegen Kartoffeln, Zuckerrüben und Runkelrüben; mengenmäßig gut, qualitativ etwas beeinträchtigt waren Getreide und Rauhfutter.

Die offene Ackerfläche erreichte 273200 ha ( +8000 ha gegenüber 1956), davon 130000 ha Getreide. Die Kartoffelernte betrug 1957: 150000 t oder 265 q/ha (1956: 1570000 t oder $266 \mathrm{q} / \mathrm{ha}$ ); die Zuckerriibenernte (schweiz. Höchstertrag) 244741 t Rüben (mittlerer Zuckergehalt $16,51 \%), 34$ oo t Zucker $(1 / 7$ des schweiz. Konsums). Die Vorarbeiten für die zweite Zuckerfabrik in der Ostschweiz wurden gefördert. Die Rebfläche ist erneut um 325 ha auf 12521 ha gesunken. Sie betrug in der deutschen Schweiz 1591 ha $(-130$ ha), in der Westschweiz (einschließlich Bielerseegebiet) 9194 ha (-150 ha) in der Südschweiz 1735 ha ( $-45 \mathrm{ha})$. Von der Gesamtfläche waren 8319 ha $(66,4 \%)$ Weißweinreben, 4202 ha $(33,6 \%)$ Rotweinreben. Der gesamte Weinertrag betrug $412864 \mathrm{hl}(-32302 \mathrm{hl})$, der mittlere Hektarertrag $33,3 \mathrm{hl}(-1,3 \mathrm{hl})$.

Die Motorisierung in der Landwirtschaft hat von 1950-1955 außerordentlich zugenommen. Motorisierte Hilfsmittel und Pferdebestand der Landwirtschaftsbetriebe:

Gesamtzahl der Traktoren, Einachstraktoren und Motormäher

Jeeps, Landrover

Aufbaumotoren auf Mähmaschinen

Bodenfräsen (mit Einschluß jener im Gartenbau)

Motorspritzen zur Schädlingsbekämpfung

Arbeitspferde, einschließlich Zuchtstuten
1939

1950 1955

$\begin{array}{ccr}15518 & 48746 & 93813 \\ ? & ? & 3201 \\ 2413 & 8704 & 8351 \\ 1323 & 4570 & 7038 \\ ? & 7424 & 11393 \\ 125400 & 114900 & 106080\end{array}$


Die Ergebnisse des Nutztierbestandes lauten:

\begin{tabular}{|c|c|c|c|c|}
\hline & \multirow[b]{2}{*}{1951} & & \multicolumn{2}{|c|}{ Veränderung } \\
\hline & & 1956 & absolut & ozentual \\
\hline Rindvieh & 1607271 & $1646229 *$ & +38958 & $+2,4$ \\
\hline Schweine & 892092 & 1161291 & +269199 & $+30,1$ \\
\hline Ziegen & 148242 & 113176 & $-\quad 35066$ & $-23,7$ \\
\hline Schafe & 191736 & 200512 & $\begin{array}{r}+8776 \\
+\quad 87\end{array}$ & $+\quad 4,5$ \\
\hline Pferde & 181374 & 116756 & -64618 & $-35,7$ \\
\hline Maultiere & 2156 & 1550 & - $\quad 606$ & -28.1 \\
\hline Esel & 393 & 370 & 一 & $-\quad 5,8$ \\
\hline Hühner & 6239881 & 6402111 & +162230 & $+2,5$ \\
\hline Gänse und Enten & 68371 & 55100 & -13271 & $-19,4$ \\
\hline Bienenvölker & 339243 & 298836 & -40407 & $-12,0$ \\
\hline
\end{tabular}

In beinahe allen Industriezweigen herrschte Hochkonjunktur. Die industrielle Tätigkeit fand indirekt in starker Bautätigkeit Ausdruck. 1957 waren 2o Kraftwerke im Bau. In 42 Städten wurden 195718384 Wohnungen (1956: 16519 ) erstellt. In den kleineren Orten war eher ein Rückgang festzustellen.

Der Außenhandel entwickelte sich 1957 wie folgt:

\begin{tabular}{lrrrr} 
& \multicolumn{2}{c}{ Einfuhr } & \multicolumn{2}{c}{ Ausfuhr } \\
& 1956 & 1957 & 1956 & 1957 \\
\hline Wagen zu 10t & 1303780 & 1328947 & 89376 & 90920 \\
Wert in Mio Fr. & 7596,9 & 8447,0 & 6203,4 & 6713,8
\end{tabular}

Defizit des Warenverkehrs: 1733,2 Mio Franken.

\begin{tabular}{|c|c|c|c|c|c|c|c|c|c|c|}
\hline & \multicolumn{4}{|c|}{ Einfuhr } & \multirow{2}{*}{$\begin{array}{c}\text { Verän- } \\
\text { derung } \\
\% / 0\end{array}$} & \multicolumn{4}{|c|}{ Ausfuhr } & \multirow{2}{*}{$\begin{array}{c}\text { Verän- } \\
\text { derung } \\
0 \%\end{array}$} \\
\hline & 1956 & $\%$ & 1957 & $\%$ & & 1956 & $0 / 0$ & 1957 & $\%$ & \\
\hline Europa & 5434,4 & 71,53 & 6100,1 & 72.21 & $+0,68$ & 3787,3 & 61,05 & 4107,3 & 61,18 & $+0,13$ \\
\hline Nordamerika & 1190,9 & 15,68 & 1346,8 & 15,94 & $+0,26$ & 872,8 & 14,07 & 893,2 & 13,31 & $-0,76$ \\
\hline Zentralamer. & 129,1 & 1,70 & 131,1 & 1,56 & $-0,14$ & 123,1 & 1,98 & 150,9 & 2,25 & $+0,27$ \\
\hline Südamerika & 289,7 & 3,81 & 295,9 & 3,50 & $-0,31$ & 382,1 & 6,14 & 413,9 & 6,17 & $+0,03$ \\
\hline Afrika & 220,9 & 2,91 & 231,0 & 2,73 & $-0,18$ & 273,4 & 4,42 & 292,8 & 4,36 & $-0,06$ \\
\hline Asien & 296,3 & 3,90 & 294,8 & 3,50 & $-0,40$ & 659,1 & 10,62 & 746,5 & 11,11 & $+0,49$ \\
\hline $\begin{array}{l}\text { Australien, } \\
\text { Ozeanien }\end{array}$ & 35,6 & 0,47 & 47,3 & 0,56 & $+0,09$ & 105,6 & 1,72 & 109,2 & 1,62 & $-0,10$ \\
\hline Total & 7596,9 & 100,00 & 8447,0 & 100,00 & - & 6203,4 & 100,00 & 6713,8 & 100,00 & - \\
\hline
\end{tabular}

Die gebietsweisen Veränderungen sind also gering.

Von der gesamten Ein- und Ausfuhr gingen 5,4 Mio t (38\%) Waren über die Basler Rheinhäfen. Insgesamt trafen 10189 Schiffe, d. h. 28 je Tag ein. Der größte Tagesverkehr war am 9. Januar mit 65 ankommenden und 60 abgehenden Schiffen. Die schweizerische Rheinund Kanalflotte hat einen Bestand von 407 Schiffen mit einer Tonnage von 328409 t.

Elektrizitätswirtschaft (Hydrographisches Jahr: 1. Oktober 1956 bis 3o. September 1957): Erzeugung und Verbrauch zeigen folgende Entwicklung:

\begin{tabular}{lccc} 
Erzcugung: & $\begin{array}{c}1955 / 56 \\
\text { in Mio kWh }\end{array}$ & $\begin{array}{c}1956 / 57 \\
\text { in Mio kWh }\end{array}$ & $\begin{array}{c}\text { Veränderung } \\
\text { in } \%\end{array}$ \\
\hline Wasserkraftwerke & 14660 & 15704 & $+7,1$ \\
Wärmekraftwerke & 235 & 190 & $-19,1$ \\
Einfuhr & 1399 & 1255 & $-10,3$ \\
\hline Total Erzeugung & 16294 & 17149 & $+5,2$ \\
\hline
\end{tabular}

* Davon 748613 Stück Braunvieh, 832 oo5 Stück Simmentaler Fleckvieh; total zusammen 96\% des Rindviehbestandes. Schwarzfleckvieh 23 753, Eringervieh 28 524, Kreuzungen 13334. 


\begin{tabular}{lccc} 
Verwendung: & $\begin{array}{c}1955 / 56 \\
\text { in Mio kWh }\end{array}$ & $\begin{array}{c}1956 / 57 \\
\text { in Mio kWh }\end{array}$ & $\begin{array}{c}\text { Veränderung } \\
\text { in } 0 / 0\end{array}$ \\
\hline Haushalt und Gewerbe & 5603 & 5997 & $+7,0$ \\
Bahnbetriebe & 1252 & 1285 & $+2,6$ \\
Industrie & 5145 & 5597 & $+8,7$ \\
Elektrokessel & 562 & 403 & $-28,3$ \\
Speicherpumpen & 215 & 184 & $-14,5$ \\
Verluste & 1720 & 1774 & $+3,1$ \\
\hline Total Inlandverbrauch & 14497 & 15240 & $+5,1$ \\
Ausfuhr & 1797 & 1909 & $+6,2$ \\
\hline
\end{tabular}

Der nasse Sommer wirkte sich in einer Steigerung der Eigenproduktion, in einer Abnahme der Einfuhr, des Einsatzes der Wärmekraftwerke und in einer Zunahme des Exportes aus.

Verkehr:

Die Bundesbahnen erhöhten ihre Transportleistungen erneut:

$$
1956
$$

1957

Zahl der beförderten Personen

215,7 Mio 24,75 Mio t

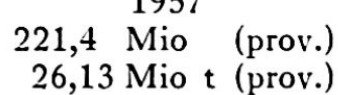

Der Straßenverkehr zeigte eine weitere Zunahme. Der Motorfahrzeugbestand am 3o. September 1957 war:

\begin{tabular}{lrrr} 
& 1956 & 1957 & $\begin{array}{c}\text { Veränderung } \\
\text { in } \%\end{array}$ \\
\hline Autobusse und Autocars & & 2594 & $+5,2$ \\
Personenwagen & 308679 & 3760 & $+12,3$ \\
Personenwagen für Warentransport & 11392 & 14037 & $+23,2$ \\
Lieferwagen & 16534 & 17495 & $+5,8$ \\
Lastwagen & 28075 & 29875 & $+6,7$ \\
Spezialwagen & 3188 & 3587 & $+12,5$ \\
Gewerbliche Traktoren & 1174 & 1106 & 5,8 \\
Motorfahrräder & 74702 & 86096 & $+22,5$ \\
Roller & 81960 & 75108 & 5,0 \\
Motorräder & 78454 & 668238 & 4,3 \\
\hline Motorfahrzeuge total & 606752 & $+10,0$ \\
\hline
\end{tabular}

Dazu kommen 2 Millionen einreisende ausländische Motorfahrzeuge. Die Planung der Autostraßen ist weiter fortgeschritten. Vorläufig sind: $385 \mathrm{~km}$ Nationalstraßen I. Klasse, $360 \mathrm{~km}$ Nationalstraßen II. Klasse, $250 \mathrm{~km}$ Alpenstraßen, $75 \mathrm{~km}$ Talstraßen. sowie Teilstrekken von städtischen Expreßstraßen vorgesehen. Finanzaufwand 2,9 Milliarden Fr.

Auch der Luftverkehr stieg erneut an: Gesamter Flugverkehr

1956

1480322

2269.5

8021

1957
1844952
25315

$\begin{array}{r}25 \\ 9 \\ \hline\end{array} 325$

Fracht in

Post in $t$

Das Liniennetz der Swissair betrug mit allet

Bevölkerung und Siedlung. Ende 1956 zählten die 3095 schweizerischen Gemeinden 5074000 Einwohner (rund 123 Einw. p. km²). Die Verstädterung schritt fort. Mit den $\mathrm{Agglo-}$ merationen sind $47,2 \%$ der Gesamteinwohnerschaft der städtischen Bevölkerung zuzurechnen (1850 lebten erst 154 ooo oder 6,4\% der rund 2,4 Mio Einwohner der Schweiz in acht Städten).

\begin{tabular}{|c|c|c|c|c|c|c|}
\hline & \multirow{2}{*}{\multicolumn{2}{|c|}{ Anzahl }} & \multicolumn{2}{|c|}{ Einwohnerzahl } & \multirow{2}{*}{\multicolumn{2}{|c|}{ Anteil in $\%$}} \\
\hline & & & 1.12 .50 & Ende 1956 & & \\
\hline & 1950 & 1956 & absolut & absolut & 1950 & 1956 \\
\hline Großstädte & 5 & 5 & 972342 & 1058600 & 20,6 & 20,9 \\
\hline Mittelstädte & $j$ & 7 & 277104 & 367500 & 5,9 & 7,2 \\
\hline Kleinstädte & 32 & 43 & 470611 & 610800 & 10,0 & 12,0 \\
\hline Städte total & 42 & 55 & 1720057 & 2036900 & 36,5 & 40,1 \\
\hline Übrige Gemeinden & 3059 & 3040 & 2994935 & 3037100 & 63,5 & 59,9 \\
\hline Schweiz total & 3101 & 3095 & 4714992 & 5074000 & 100,0 & 100,0 \\
\hline
\end{tabular}

Quellen: Die Volkswirtschaft 1957/58, Berichte der Schweiz. Bankgesellschaft, der Schweiz. Kreditanstalt, Bulletin des SEV 1957, Energie-Konsument 1957, führende Tageszeitungen u. a.m. Ferner sei zahlreichen eidgenössischen und kantonalen Ämtern für bereitwillige Auskunft bestens gedankt. 